\title{
Body Composition Characteristics of a Load- Capacity Model: Age-Dependent and Sex-Specific Percentiles in 5- to 17-Year-Old Children
}

\author{
Isabel Gätjens ${ }^{a} \quad$ Steffen Christian Ekkehard Schmidt ${ }^{b} \quad$ Sandra Plachta-Danielzik ${ }^{c}$ \\ Anja Bosy-Westphal ${ }^{a}$ Manfred James Müller ${ }^{a}$ \\ anstitute of Human Nutrition and Food Science, Christian-Albrechts University Kiel, Kiel, Germany; ${ }^{\text {b Institute }}$ \\ of Sports and Sports Science, Karlsruhe Institute of Technology, Karlsruhe, Germany; ${ }^{C}$ Kompetenznetz \\ Darmerkrankungen e.V., Kiel, Germany
}

\section{Keywords}

Body composition - Fat mass index - Fat-free mass index .

Load-capacity model · Children

\begin{abstract}
Introduction: Body composition assessment is superior to the use of body mass index (BMI) to characterize the nutritional status in pediatric populations. For data interpretation, suitable reference data are needed; hence, we aimed to generate age-dependent and sex-specific body composition reference data in a larger population of children and adolescents in Germany. Methods: This is a cross-sectional study on a representative group of 15,392 5- to 17-year-old children and adolescents. Body composition was assessed by bioelectrical impedance analysis using a population-specific algorithm validated against air displacement plethysmography. Age- and sex-specific percentiles for BMI, fat mass index (FMI), fat-free mass index (FFMI), and a "load-capacity model" (characterized by the ratios of fat mass [FM]/ fatt-free mass [FFM] and $F M / F F M^{2}$ ) were modeled using the LMS method. Results: BMI, FMI, FFMI, FM/FFM, and FM/FFM ${ }^{2}$ curves showed similar shapes between boys and girls with steady increases in BMI, FMI, and FFMI, while FM/FFM²-cen-
\end{abstract}

karger@karger.com www.karger.com/ofa

Karger $\stackrel{\text { ' }}{5}$

GOPEN ACCESS
(C) 2021 The Author(s)

Published by S. Karger AG, Basel

This is an Open Access article licensed under the Creative Commons Attribution-NonCommercial-4.0 International License (CC BY-NC) (http://www.karger.com/Services/OpenAccessLicense), applicable to the online version of the article only. Usage and distribution for commercial purposes requires written permission. tiles decreased during early childhood and adolescence. Sex differences were observed in FMI and FM/FFM percentiles with increases in FMI up to age 9 years followed by a steady decrease in FM/FFM during and after puberty with a fastgrowing FFMI up to age 17 in boys. The prevalence of low FFM relative to $\mathrm{FM}$ reached more than $60 \%$ in overweight children and adolescents. Conclusion: These pediatric body composition reference data enable physicians and public health scientists to monitor body composition during growth and development and to interpret individual data. The data point out to an early risk of sarcopenia in overweight children and adolescents.

(c) 2021 The Author(s).

Published by S. Karger AG, Basel

\section{Introduction}

The shortcomings of height- and weight-based standards to characterize the nutritional status and its related health risks in children are well known $[1,2]$. Although body mass index (BMI) and percentage fat mass (\% FM) are still used to define overweight and obesity, they are incomplete because they do not address body proportions and "functional body mass", for example, fat-free 
mass (FFM) and its relation to FM. The failure of BMI and \% FM to account for the contributions of FM and FFM to body weight may over- and underestimate obesity in subjects with high and low FFM and, thus the BMIassociated metabolic and cardiovascular risks. In addition, BMI cannot address the loss of FFM in children with chronic diseases, which is associated with their prognosis (see [3-6]).

FM and FFM have to be normalized for height because: (i) absolute values of either FM or FFM do not identify issues of malnutrition in subjects of different constitutions, (ii) \% FFM may increase rather than decrease with weight loss and, thus may not reflect its specific loss and (iii) dividing FM by body weight (which includes FM) results in relatively small changes in \% FM with changes in weight and adipose tissue mass $[1,2,7]$. To overcome those issues, fat mass index (FMI) and fat-free mass index (FFMI) are considered superior to BMI and \% FM to assess the nutritional status of children and adolescents [1, 4, 7-9]. These indices include body size for normalization and address FM and FFM independently from each other, which give rise to identify specific phenotypes with the same BMI but differing in FMI and FFMI $[1,10]$.

More recently, the dynamic and independent inter-relationships and, thus inter-active effects of FMI and FFMI have been identified as specific determinants of disease risk prediction $[11,12]$. For example, a high FMI at low FFMI partly characterizes a sarcopenic obese patient characterized by a decline in muscle mass and function. This concept refers to the traits of "metabolic load" (e.g., high fat mass and obesity) and "metabolic capacity" (e.g., organs and tissues within FFM related to maintenance of metabolic homeostasis) [1].

Since (i) the identification of specific body composition phenotypes is presently under-investigated in pediatric populations and (ii) there are only few body composition reference curves (which were obtained from relatively small cohorts of children and adolescents using advanced multi-component models like the 4-compartment model in 533 children by Wells et al., 2012 [8], or the combined use of DXA, deuterium dilution and total body potassium in 1,122 children by Shypailo and Wong [9], respectively), we hereby provide normative data for FMI, FFMI, and FM/FFM-ratio in a large and representative group of 15,392 5-17-year-old Caucasian children and adolescents in Germany. The pediatric reference data on a "load-capacity model" can be extended into adulthood [12] and, thus will add to improve our understanding of the long-term impact of birth weight, malnutrition, and growth disorders as well as to interpret nutritional status in clinical practice. Our present analysis adds to a previous study on pediatric reference data of \% FM obtained from a multi-cohort population of Germany [13].

\section{Materials and Methods}

\section{Study Population}

Cross-sectional data were collected as part of the Kiel Obesity Prevention Study (KOPS). KOPS has been started since 1996 as a cross-sectional and longitudinal study in Kiel. To date, more than 15,500 children and adolescents between 5 and 17 years have been investigated and partly reinvestigated in randomly sampled primary and secondary schools in different neighborhoods of Kiel in the 1st school grade (5-7 years old), 4th school grade (9-12 years old) and 8th school grade (13-16 years old) [14]. KOPS include 3 cross-sectional studies and 1 longitudinal study. Between 1996 and 2001, 4,997 5- to 7-year-old children (1st grade, cohort 1) were recruited that were $41 \%(n=12,254)$ of the total population who entered the school-entry examinations in this time period in Kiel. Between 2000 and 2005, 4,487 children aged 9-11-years were enrolled (4th grade, cohort 2) and between 2004 and 2009, 5,767 data were collected from 13- to 16-year-old adolescents. Because of the same total population of all 3 cohorts, there were subgroups of children who were included in 2 or 3 cohorts. Four years after enrollment, 1,671 of the 9-11-year old children were reinvestigated (35\% of cohort 1). A 2 nd group with follow-up at 4 years consisted of the intersection of cohorts 2 and 3, which included 1,453 children. Eight years after enrollment, 1,192 were reinvestigated (24\% of cohort 1$)$. A subgroup $(n=742)$ participated at all 3 time points. KOPS intends to characterize the determinants and long-term outcome of childhood overweight and the effect of early preventive measures against excessive weight gain within schools and families. Participation was voluntary and without any eligibility criteria except the willingness to participate and a written informed consent from the parents. The study was approved by the local Ethical Committee of the Medical Faculty of the Christian-Albrechts University (Kiel, Germany). More details of the aims, study design, recruitment procedure, and representativity of the study population are described elsewhere [15-17]. A population of 15,392 children and adolescents aged 5-17 years was used to generate percentiles for BMI and variables of body composition.

\section{Weight Status and Body Composition}

Anthropometric measurements of height and weight at the age of 5-17 years were performed by trained observers from the Institute of Human Nutrition and Food Science, Christian-Albrechts University in Kiel following standard procedures. Body weight was recorded to the nearest $0.1 \mathrm{~kg}$ using a calibrated digital scale (Model 861, seca GmbH, Hamburg, Germany) with subjects wearing light underwear. Body height was assessed to the nearest $0.1 \mathrm{~cm}$ using a portable stadiometer (Model 214, seca GmbH, Hamburg, Germany). BMI was calculated from weight and height as $\mathrm{kg} / \mathrm{m}^{2}$. Body composition was assessed by bioelectrical impedance analysis (BIA), using a tetrapolar BIA device with hand to foot electrodes (BIA 2000-C; data Input GmbH, Darmstadt, Germany). FFM was calculated using a BIA algorithm generated and crossvalidated for children and adolescents in our laboratory using air displacement plethysmography as a reference: FFM $[\mathrm{kg}]=0.66 \times$ 
[height $\mathrm{t}^{2}\left(\mathrm{~cm}^{2}\right) /$ resistance $\left.(\Omega)\right]+0.196 \times$ weight $[\mathrm{kg}]+0.157 \times$ reactance $[\Omega]+0.348 \times$ age [years] $-12,083$ [13]. FM was calculated from the difference between body weight and FFM. FM and FFM were normalized by height ${ }^{2}$ to FMI and FFMI. More recently, we have validated BIA data against results obtained by either the 4-compartment model [18] or whole-body magnetic resonance imaging [19]. For public health research on pediatric population, it has been shown that when compared with a reference method, BIA data have negligible average bias but wider limits of agreement [1].

\section{Pubertal Stage}

Pubertal development (male genitalia development, female breast, and pubic hair growth in boys and girls) was self-estimated by the 13-17-year-old adolescents using standardized pictures of Tanner staging on scales from II to V. Up to the age of 12 years, pubertal stage was assumed to be prepubertal (scale I).

\section{Age- and Sex-Specific Body Composition Centiles}

Age- and sex-specific percentiles for BMI, FMI, FFMI, FM/ FFM, and FM/FFM ${ }^{2}$ were modeled using the LMS method [20]. Respective percentiles are summarized by 3 curves representing the skewness (or the Box-Cox-power L), the median (M), and the coefficient of variation as quantities that change with age, plotted as sex-specific smooth curves versus age. For generating percentiles, data obtained in our reference population were normalized by dividing the age into groups and then applying a power transformation, which extends 1 tail of the distribution and contracts the other, eliminating skewness in the variable under analysis. A curve is fitted to the normalizing power transformation for each age-group, generating an optimum "L" (skewness) curve that normalizes the dependent measure (BMI, FMI, FFMI, FM/FFM, and $\mathrm{FM} / \mathrm{FFM}^{2}$ ) over the entire age range. The same procedure was performed for curves representing $\mathrm{M}$ and $\mathrm{S}$, in each case separately for boys and girls. Starting from L $11 / 4, M 1 / 45$, and S $1 / 43, \mathrm{~L}, \mathrm{M}$, and S were increased by 1 and a new model was run until change in deviance between 2 models were below ten units. The age- and sexspecific percentile curves for BMI, FMI, FFMI, FM/FFM, and FM/ FFM $^{2}$ were 3rd, 10th, 25th, 50th, 75th, 90th, and 97th.

\section{Statistical Analysis}

Statistical analyses were performed with IBM ${ }^{\circledR}$ SPSS ${ }^{\circledR}$ Statistics for Windows (version 26.0 Somers, NY, USA). Descriptive statistics of the study population, stratified by sex and age-groups are presented. Mann-Whitney U-test was done to determine significant differences between boys and girls. The level of significance was set at $p<0.05$ (two-sided). Age- and sex-specific percentile curves for BMI, FMI, FFMI, FM/FFM, and FM/FFM ${ }^{2}$ were modeled using LMS Chartmaker Pro (V. 2.3) curve fitting procedure by Cole and Green [20].

\section{Results}

Characteristics of the reference population used to generate the percentiles are given in Table 1. The group was stratified according to 3 age-groups and sex. Prevalence of overweight and obesity were similar in all age-

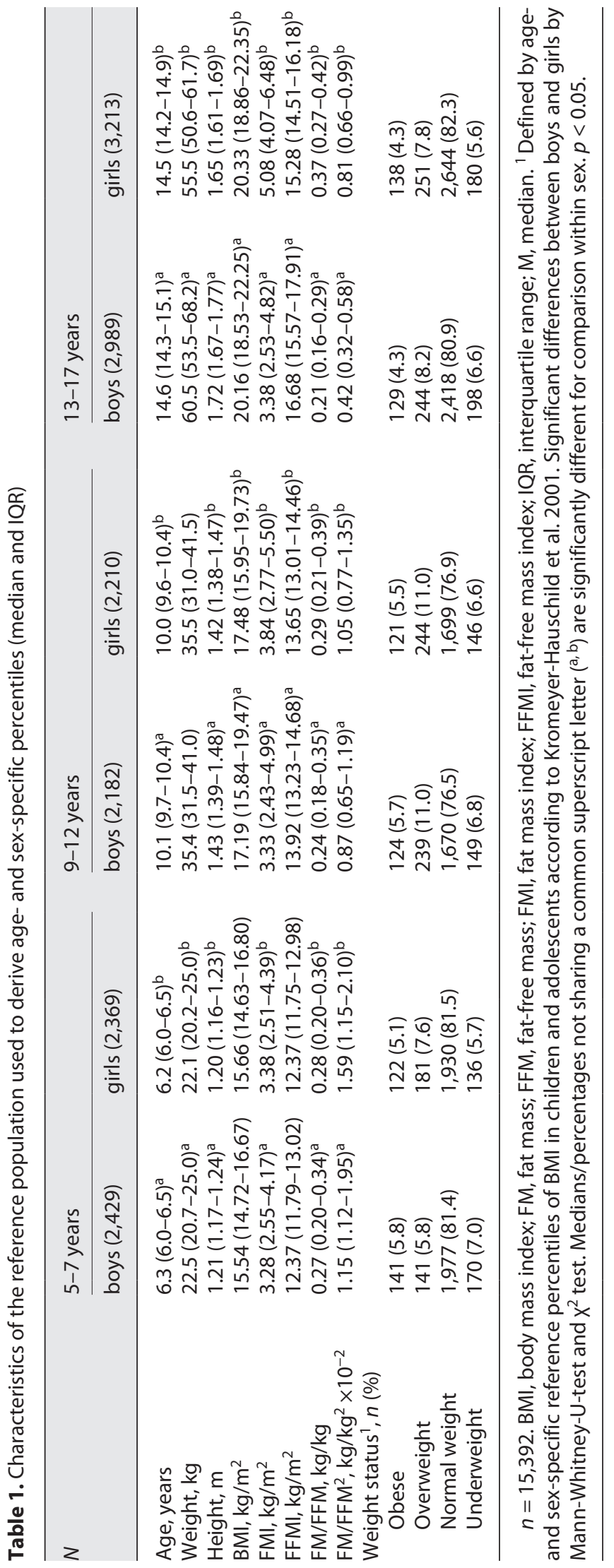

Obes Facts 2021;14:593-603 

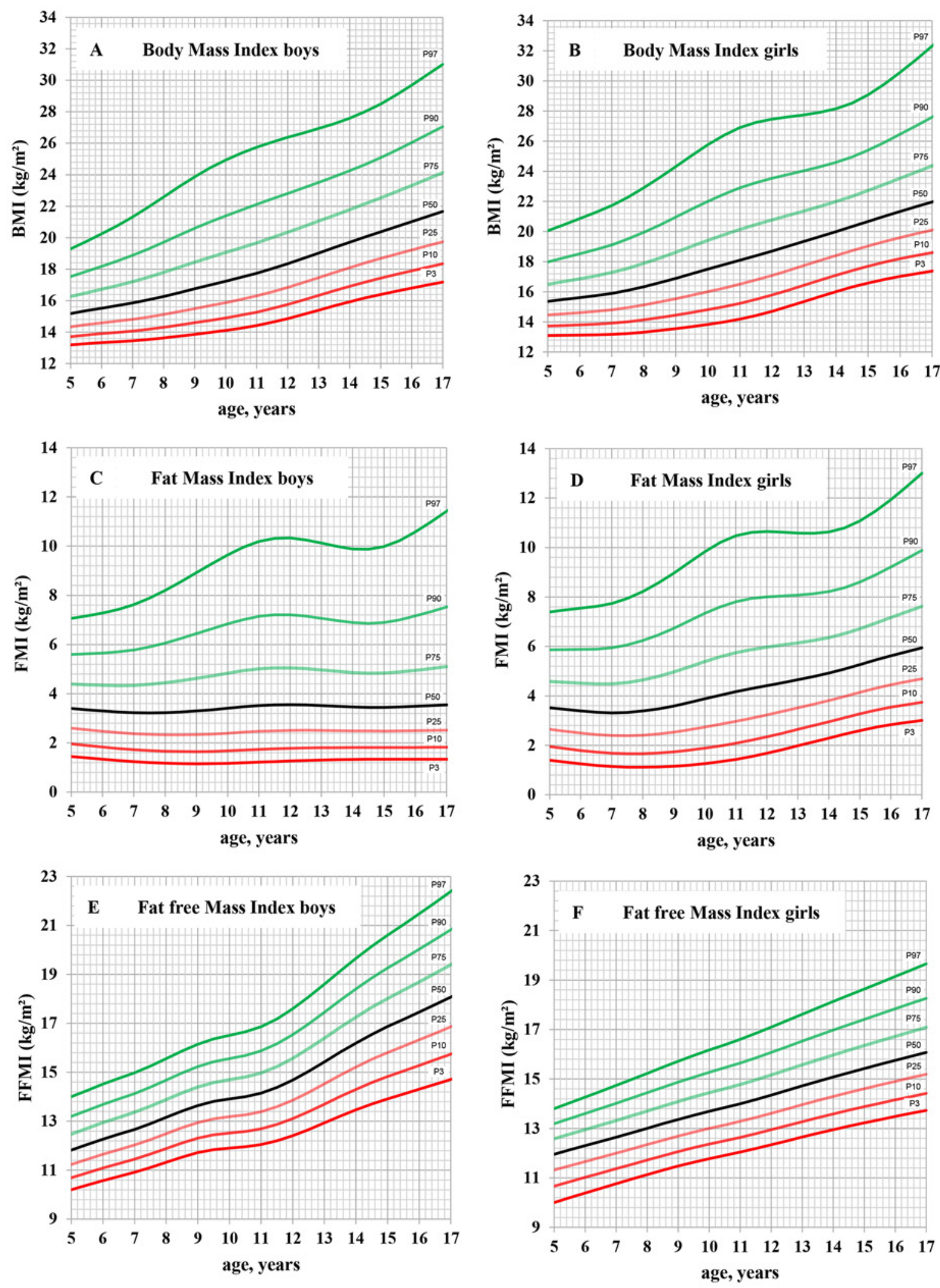

(Figure continued on next page.) 


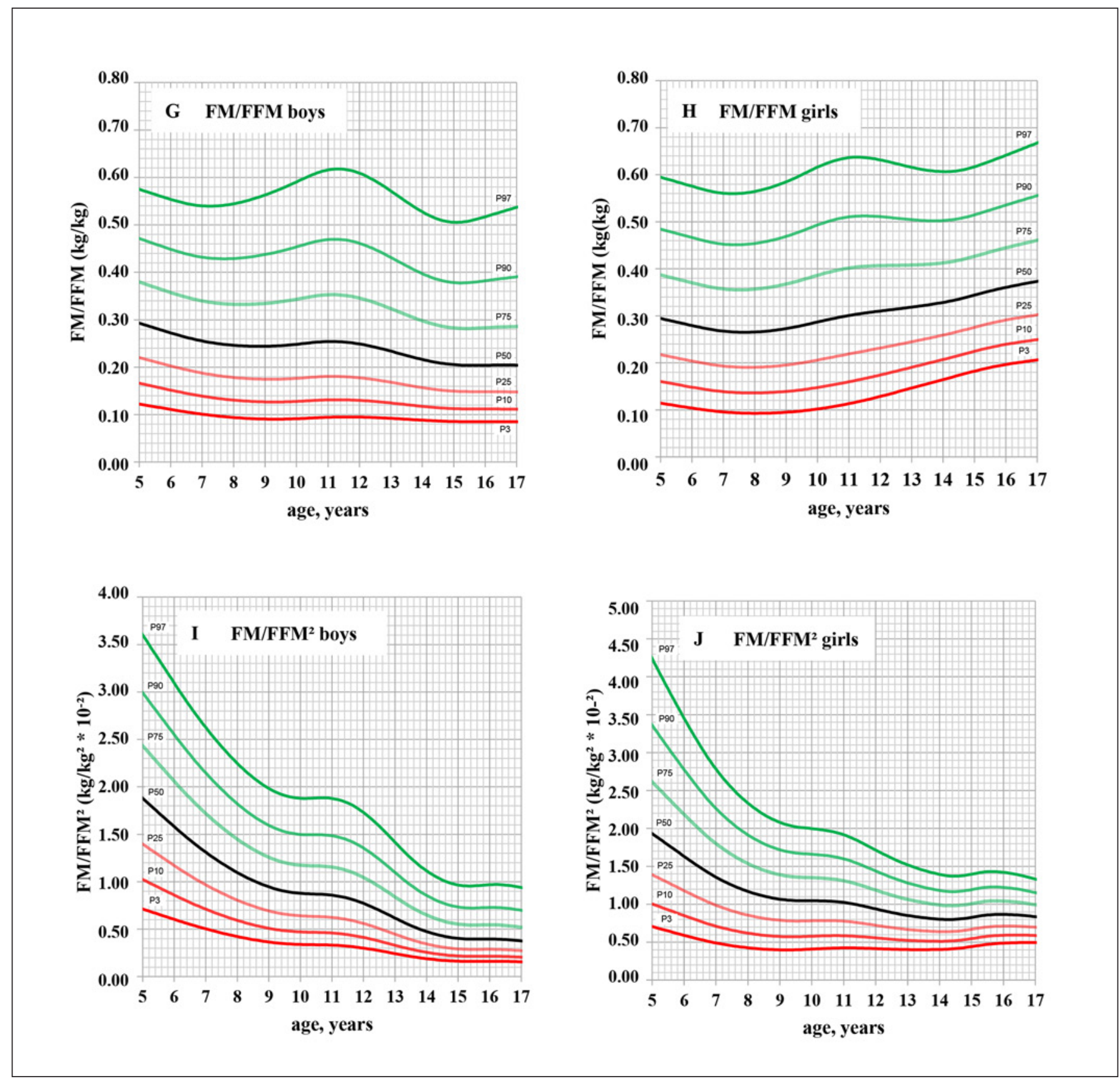

Fig. 1. A-J Age- and sex-specific percentile curves of BMI, FMI, FFMI, FM/FFM and FM/FFM ${ }^{2}$ in the reference population of 15,392 5- to 17-year-old children and adolescents in Germany. FM, fat mass; BMI, body mass index; FMI, fat mass index; FFMI, fat-free mass index; FFM, fat-free mass.

groups. BMI, FMI, and FFMI increased with age with significant differences between boys and girls in each agegroup except for a similar BMI, FMI, and FFMI in 5-7-year-old boys and girls. Children aged 5-7 and 9-12 years were all at prepubertal stage (Tanner stage I). Most of the 13-17-year old adolescents were in Tanner stage III (25.5\%) and IV (59.1\%). Compared to girls, more boys were in Tanner stage II and III (Tanner stage I: 3.3\% vs. $2.2 \%, p<0.05$; Tanner stage II: $31.2 \%$ vs. $20.9 \%, p<0.05$ ), whereas more girls were classified in Tanner stage IV and 


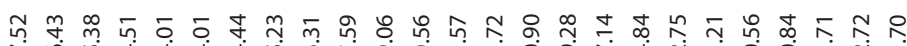
ఫ 守守守守 กิ น बิ山

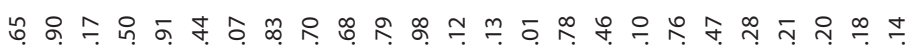

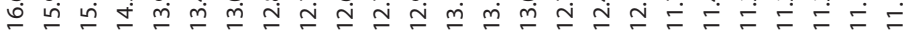
స 숭 字

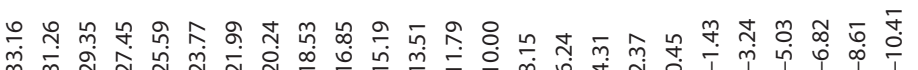
लंख

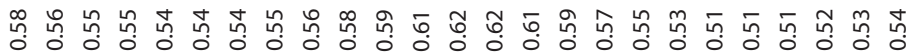

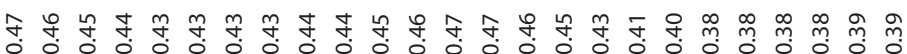

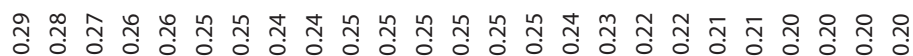

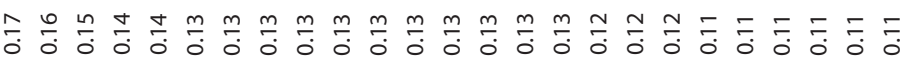

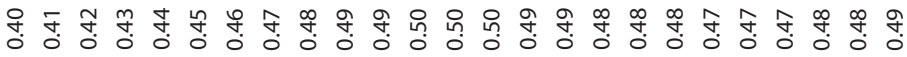

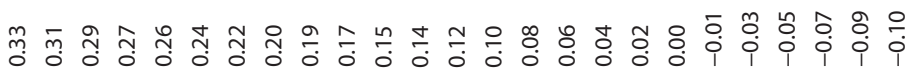

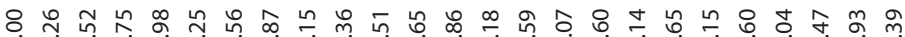

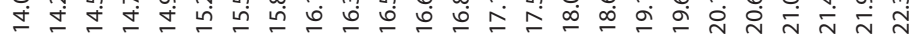

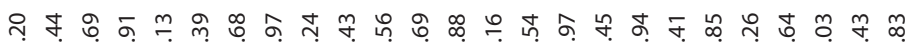

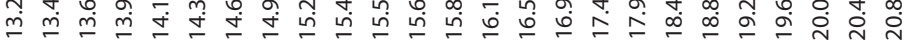

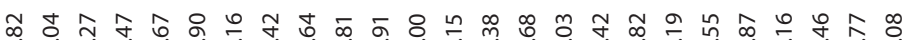

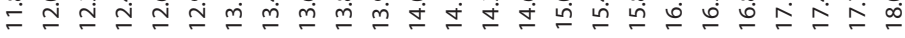

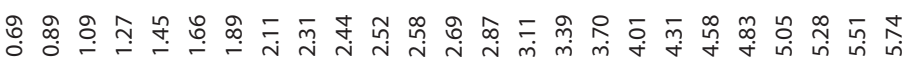
$\sim F=F F \simeq \simeq \simeq \simeq \simeq$

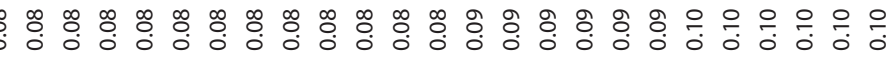
œ i i i

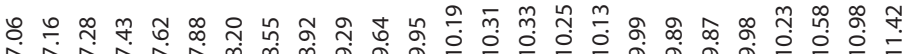

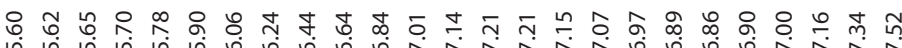

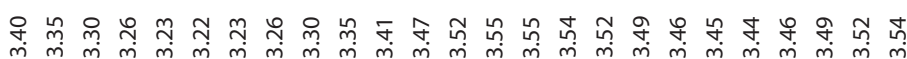

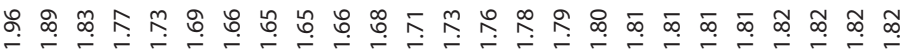

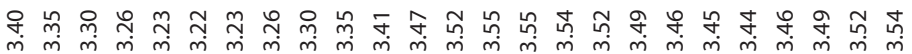

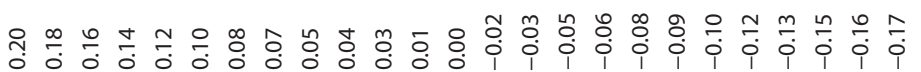

\begin{tabular}{|c|c|c|}
\hline & $\hat{a}$ & 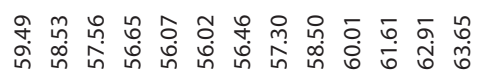 \\
\hline & \& & 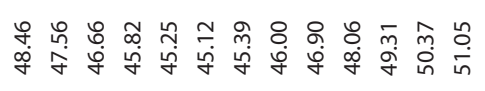 \\
\hline & 员 & 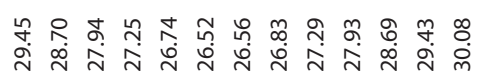 \\
\hline & 음 & 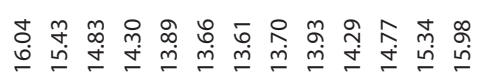 \\
\hline$\frac{\sqrt{2}}{2}$ & $\backsim$ & 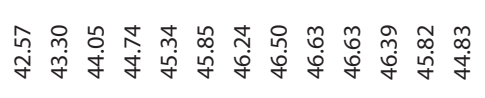 \\
\hline & - & 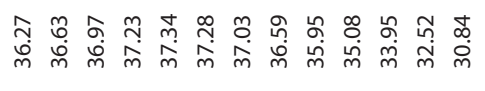 \\
\hline & à & 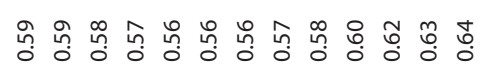 \\
\hline & ஃ & 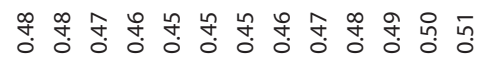 \\
\hline & $\ddot{n}$ & 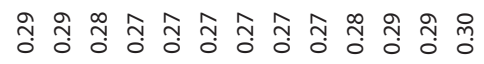 \\
\hline & $\frac{\circ}{2}$ & 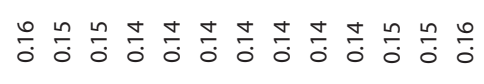 \\
\hline 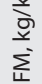 & $\backsim$ & 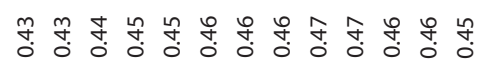 \\
\hline$\sum_{i=}^{\frac{1}{4}}$ & - & 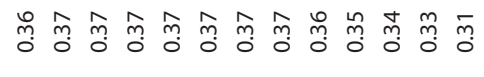 \\
\hline & $\hat{a}$ & 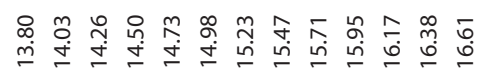 \\
\hline & ஃ & 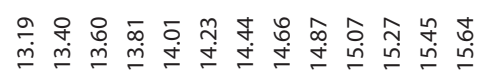 \\
\hline & 응 & 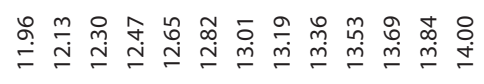 \\
\hline & $\frac{}{2}$ & 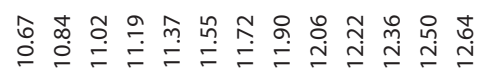 \\
\hline 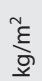 & $\backsim$ & 足 : \\
\hline$\sum_{\psi}^{\bar{\psi}}$ & - & 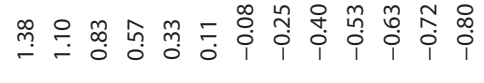 \\
\hline & $\hat{a}$ & 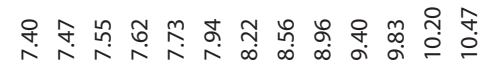 \\
\hline & ஃ & 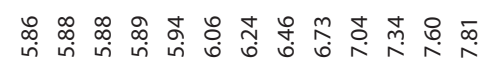 \\
\hline & ํํㅇ & 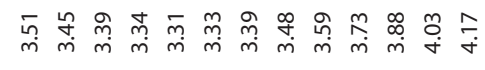 \\
\hline & $\div$ & 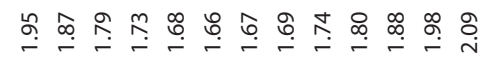 \\
\hline$\underset{\underline{g}}{\tilde{E}}$ & $\backsim$ & 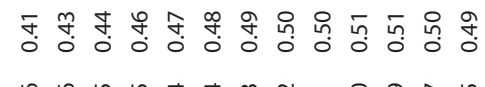 \\
\hline$\sum_{u}^{*}$ & \lrcorner & ָ̦: \\
\hline$\stackrel{n}{=}$ & $\overrightarrow{0}$ & \\
\hline
\end{tabular}




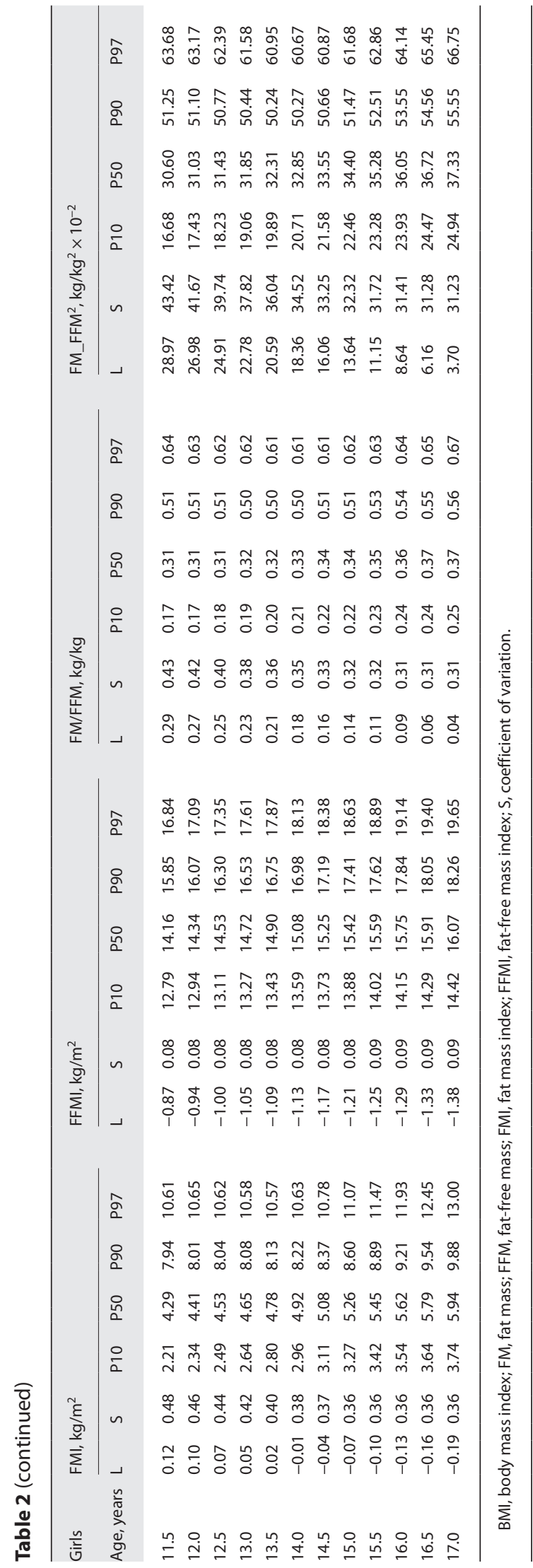

Body Composition Percentiles in Children and Adolescents
V (Tanner stage IV: $61.0 \%$ vs. $57.0 \%, p<0.05$; Tanner stage V: $15.9 \%$ vs. $8.4 \%, p<0.05)$.

Sex-specific percentiles for BMI, FMI, FFMI, FM/ FFM, and FM/FFM ${ }^{2}$ are shown in Figure $1 \mathrm{~A}-\mathrm{J}$ and Table 2. BMI, FMI, FFMI, FM/FFM, and FM/FFM ${ }^{2}$ curves showed similar shapes between boys and girls with a steady increase for BMI, FMI, FFMI, and FM/FFM (in girls only) and a steady decrease for $\mathrm{FM} / \mathrm{FFM}^{2}$ curves during early childhood and adolescence. Sex differences were observed in FMI (Fig. 1C, D; Table 2) and FM/FFM percentiles (Fig. 1G, H; Table 2), with girls showing a slight decline up to the age of 9 years, followed by a slow increase, whereas percentile curves in boys steadily increased (FMI percentiles) or slightly decreased (FM/FFM percentiles) throughout the whole age range between 5 and 17 years. FFMI and FM/FFM ${ }^{2}$ percentiles showed a consistent increase or decrease in girls (Fig. 1F, J; Table 2), whereas boys showed a plateau (lower percentiles) or only a minimal increase in FFMI or decrease in FM/ $\mathrm{FFM}^{2}$ (higher percentiles) from 9 to 11 years, followed by a relatively fast-growing increase in FFMI or decrease in FM/FFM ${ }^{2}$ up to the age of 17 years (Fig. 1E, I; Table 2).

Figure 2 shows the distribution of overweight children and adolescents according to their FM/FFM-ratio. When compared with the 90th FM/FFM percentile, the prevalence of a relatively low FFM (e.g., a high FM/FFM-ratio as defined by values exceeding the 90th percentile) was about $60 \%$ in boys (Fig. 2A) and in girls (Fig. 2B).

\section{Discussion}

In infants and children, body composition reference data have already replaced BMI charts (e.g., $[8,21,22])$. The present standards in the assessment of the nutritional status and body composition have been published in 2014 [21]. Although widely used BMI still is a global proxy of nutritional status. Conceptually, BMI has limitations, for example, it is related to \% FM which cannot be used for inter-individual comparisons and thus has been replaced by FMI [7], and dividing fat mass by weight (as done in \% FM) fat is present in both nominator and the denominator [21]. In addition, BMI cannot be used to compare children from different ethnic groups and children with specific diseases. All these are arguments against the BMI (thus, arguing against an ongoing use of the BMI is a strong argument for alternative measures). To overcome the limitations of the BMI, throughout the last 2 decades, numerous body composition reference data for children and adolescents using different methods 


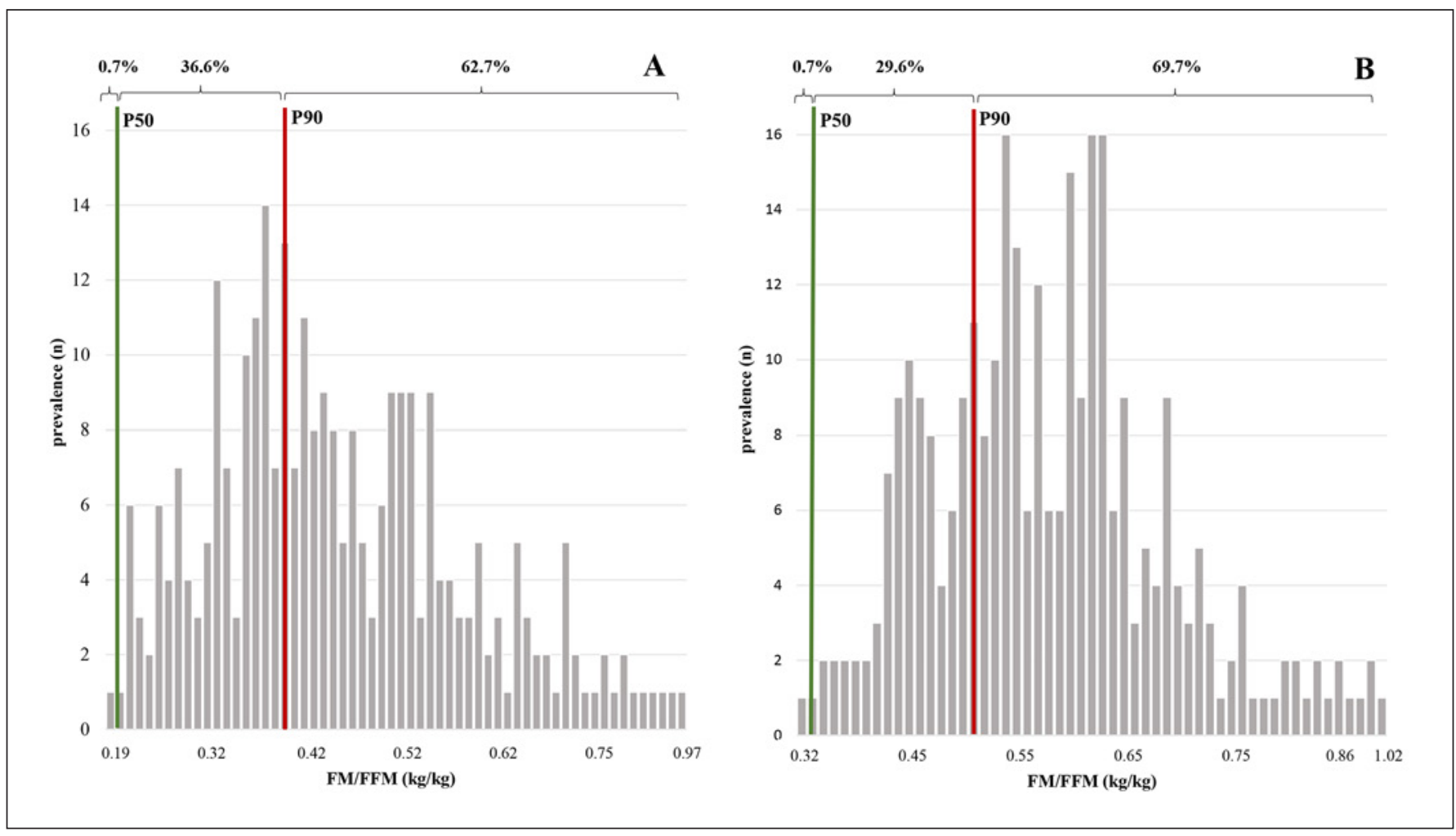

Fig. 2. A, B Prevalence rates of FM/FFM $(\mathrm{kg} / \mathrm{kg})$ in overweight and obese boys $(\mathbf{A} ; n=286)$ and girls $(\mathbf{B} ; n=297)$ at age 14-17 years defined by BMI $>90$ th percentile of our own percentiles of the reference population of 15,392 5-17-year-old children and adolescents in Germany. Prevalences are indicated according to $\leq 50$ th, between $>50$ th and $\leq 90$ th percentile and $>90$ th percentile of FM/FFM. FM, fat mass; FFM, fat-free mass; BMI, body mass index; FMI, fat mass index; FFMI, fat-free mass index; FM, fat mass; FFM, fat-free mass.

(including both, so-called "field" methods or techniques and gold standard methods using multi-component models) and different populations have been published (see Table 1 in [21]). These data can now be used "to discern and interpret the physiological basis of secular trends in nutritional status and to allow body composition to be monitored and managed in clinical practice" [21]. We agree that presently this is not everyone's knowledge (but it should become so). Accordingly, body composition reference data have been included into the official guidelines defining overweight/obesity and malnutrition [23].

In a previous article we had published LMS centiles for $\%$ FM of a representative cohort of German children and adolescents [24]. When compared with \% FM, FMI or FFMI are the height-normalized variables which improve the assessment of body composition as indicators of the nutritional status [4, 7-9]. Additionally, the ratio of FM/ FFM (or FM/FFM ${ }^{2}$ ) describes the relationship between metabolic loads accumulated by lifestyle and body function and thus could better indicate its impact on health than FM alone [25]. Since growth rates during infancy and childhood are linked to metabolic homeostasis and health outcomes in later life, data on body composition trajectories are needed to follow tissue accretion and, thus, the partitioning of weight into FM and FFM, during development and throughout changes in height and weight [22]. Our present reference data describe sex differences and age-depend periods of FM and FFM accretion (Fig. 1C-F; Table 2) as well as of the "metabolic load" to "metabolic capacity"-traits, which is reflected by the FM/FFM and FM/FFM ${ }^{2}$-ratios (Fig. 1G-J; Table 2) [26]. We may suggest that a sarcopenic obese phenotype (i.e., thin-fat-overweight) could be defined as $>90$ th percentile of FM/FFM or FM/FFM ${ }^{2}$.

Our normative data on FMI and FFMI in children add to respective population data obtained from BIA measurements in adults (e.g., published by $[12,27])$. However, looking separately at FMI or FFMI may lead to misinterpretation because the relationship between the 2 compartments, and thus the concept of the "capacity-load model" 
is not taken into account $[1,10,28]$. The "capacity-load model" is a conceptional model that was developed to improve the understanding of the life-course etiology of metabolic diseases [26]. "Metabolic capacity" refers to physiological traits strongly influenced by factors like nutrition and growth during early development during pregnancy and infancy, which promote the long-term capacity for metabolic homeostasis and health outcomes later in life while "Metabolic load" refers to components of lifestyle and nutritional status that challenges the long-term capacity of metabolic homeostasis and thus disease risk [12, 26]. Addressing the conceptual "capacity-load model" instead of individual body composition components allows insights into metabolic and physical functioning including perturbations and health risks due to the relationship between individual body composition components, for example, a "high fat-to-lean ratio" suggests a high metabolic load for a given BMI, which increases the risk of noncommunicable diseases $[1,10-12,27,28]$.

When compared with previous body composition reference data for children, the present data addressed the relationship between FM and FFM, which is expressed by the ratios of either FM to FFM or FM to FFM ${ }^{2}$ (Fig. 1G-J). Sex- and BMI-specific references of body composition indices of the "capacity-load model" have been already published for adults from age 18 years to older individuals $>60$ years excluding very tall and morbidly obese subjects from the 1999-2004 NHANES population [12]. These data were taken as a suitable measure of body composition phenotypes (e.g., "sarcopenic obese phenotype" characterized by a low muscle mass and function in obese children or "thin-fat phenotype" of normal weight patients with a low lean mass and a high percentage of FM $[1,29,30])$ and risk stratification assuming that risk results from the relative contribution of the 2 body components. For example, a high BMI may camouflage a low FFM in the case of "sarcopenic obesity." Using the NHANES data, sarcopenic obesity was defined pragmatically, for example, by an FMI > the 50th population-specific centile associated with a lean mass index below the respective P50 resulting in its prevalence of 14.7 and $22.9 \%$ of obese women and men, respectively [11]. In our 1 st analysis, we had applied the P50 of FM/FFM compared to Prado et al. [11]. Analogous to the classification of overweight, we considered the P90. Although we have used the 90th instead of the 50th centile of the FM/FFMratio the prevalence of a relatively low FFM was high in overweight children and adolescents (Fig. 2A, B). These findings point out to a possible early risk of sarcopenia in overweight children and adolescents.

Body Composition Percentiles in Children and Adolescents
Our present LMS centiles of the ratios of FM/FFM and FM/FFM ${ }^{2}$ obtained in children and adolescents add to previous data in adults [12]. The ratio of FM/FFM can overestimates FM in those with greater FFM, thus the best assessment for whole-body fatness and the most appropriate index was found to be FM/FFM ${ }^{2}$ [31]. These normative data may provide a suitable basis to define different phenotypes, which may impact growth and development as well as nutritional status-related metabolic disturbances and later health risks. Thus, our reference data can be used to identify specific body composition phenotypes of children and adolescents (e.g." "sarcopenic obese phenotype" or "thin-fat phenotype" $[10,11])$. Since metabolic and functional capacity of the body is determined early in life and the majority of children tend to track along their given centile, childhood overweight and presumably early manifestation of a sarcopenic obese phenotype may persist into adulthood [21, 32, 33]. In children and adolescents, reference data of indices of the "capacity-load model" thus add to a 1st risk assessment of metabolic and physical perturbations and growth disorders. In addition, assessing FMI and FFMI independently and its inter-relationship are useful for children with chronic diseases. FMI and FFMI may be affected differently in chronic disease, and a normal BMI may then camouflage a reduced FFM. For example, sarcopenic obesity has been identified as a phenotype of malnutrition in chronically ill children, for example, in survivors of pediatric allogeneic hematopoietic stem-cell transplantation, juvenile rheumatoid arthritis, end-stage renal disease, after liver transplantation, and Crohn's disease [1, 4-6]. Additionally, the "thin-fat phenotype" (also described as normal weight obesity, metabolic obesity, metabolically unhealthy nonobese) is found to be very common in South Asian countries, like Indian population and associated with a high cardio-metabolic risk, which is similar to individuals with overt obesity $[30,34]$.

There are some limitations of the study that have to be mentioned. Up to the age of 12 years, pubertal stage was assumed to be prepubertal, because Tanner stages were only queried in adolescents. The mean age at menarche for girls in Europe is about 12 years and followed at least 1 -year pubertal development. Compared to the representative prevalence rates of overweight and obesity of the German Health Interview and Examination Survey for Children and Adolescents (KiGGS; 15, 4\% for boys and girls respectively, [35]), our population had lower prevalence rates of overweight and obesity with $13,4 \%$ for boys and $13,4 \%$ for girls. The possible value and the limitations of BIA have been discussed in detail [21]. As has been 
mentioned there "For public health research, BIA and skinfold thicknesses show negligible average bias but have wider limits of agreement than specialized techniques such as DXA, air displacement plethysmography, and isotope dilution for measurement of TBW. Given the range of options now available, and evidence for the degree of agreement in SDS between methods, these body composition growth charts may therefore aid in monitoring individuals and populations over time." In our hands, we have already validated BIA against 2 gold standard methods, that is, the 4-compartment model as well as whole-body MRI $[36,37]$. In the former study, "Population specificity is of minor importance when compared with discrepancies between different reference methods." At least, our results showed that the BMI percentiles in adolescents exceed the global adult data, which have to be mentioned.

To summarize, these pediatric reference data will add to clinical body composition assessment by enabling an interpretation of individual data. They perform a suitable basis for future public health research and clinical practice.

\section{Acknowledgments}

There are no acknowledgments to declare.

\section{Statement of Ethics}

Parents of the subjects provided their written informed consent. The study was approved by the local Ethical Committee of the Medical Faculty of the Christian-Albrechts University (Kiel, Germany; AZ: A 36/95).

\section{Conflict of Interest Statement}

The authors have no conflicts of interest to declare.

\section{Funding Sources}

KOPS was supported by the Deutsche Forschungsgemeinschaft (DFG Mü 5.1, 5.2, 5.3 and 5.5; DFG PL781/2-1), Kompetenznetz Adipositas (Competence Network Obesity) funded by the Federal Ministry of Education and Research (FKZ: 01GI1121A). We acknowledge financial support from Land Schleswig-Holstein within the funding programme Open Access Publikationsfonds. The sponsors of the study had no role in the design, analysis, or writing of the paper. The corresponding author had full access to all the data in the study and had final responsibility for the decision to submit for publication.

\section{Author Contributions}

M.J.M. developed the aim of the study; I.G. and S.C.E.S. did the statistical analyses; I.G., M.J.M., and A.B.W. interpreted the data; I.G., M.J.M. and A.B.W. wrote the final draft of the article. All authors discussed the data and approved the final version of the article. A.B.W. will act as guarantor for the article.

\section{Data Availability Statement}

All data generated or analyzed during this study are included in this article. Further inquiries can be directed to the corresponding author.

\section{References}

1 Wells JCK. The metabolic ghetto: an evolutionary perspective on nutrition, power relations and chronic diseases. [Internet]. Cambridge: Cambridge University Press; 2016.

2 Müller MJ, Braun W, Enderle J, Bosy-Westphal A. Beyond BMI: conceptual issues related to overweight and obese patients. Obes Facts. 2016;9(3):193-205.

3 Thayu M, Denson LA, Shults J, Zemel BS, Burnham JM, Baldassano RN, et al. Determinants of changes in linear growth and body composition in incident pediatric Crohn's disease. Gastroenterology. 2010;139(2):4308.

4 Weber DR, Moore RH, Leonard MB, Zemel BS. Fat and lean BMI reference curves in children and adolescents and their utility in identifying excess adiposity compared with BMI and percentage body fat. Am J Clin Nutr. 2013;98(1):49-56.
5 Lara-Pompa NE, Hill S, Williams J, Macdonald S, Fawbert K, Valente J, et al. Use of standardized body composition measurements and malnutrition screening tools to detect malnutrition risk and predict clinical outcomes in children with chronic conditions. Am J Clin Nutr. 2020;112(6):145667.

6 Hron BM, Duggan CP. Pediatric undernutrition defined by body composition - are we there yet? Am J Clin Nutr. 2020;112(6):14246.

7 VanItallie TB, Yang MU, Heymsfield SB, Funk RC, Boileau RA. Height-normalized indices of the body's fat-free mass and fat mass: potentially useful indicators of nutritional status. Am J Clin Nutr. 1990;52(6): 953-9.

8 Wells JC, Williams JE, Chomtho S, Darch T, Grijalva-Eternod C, Kennedy K, et al. Body- composition reference data for simple and reference techniques and a 4-component model: a new UK reference child. Am J Clin Nutr. 2012;96(6):1316-26.

9 Shypailo RJ, Wong WW. Fat and fat-free mass index references in children and young adults: assessments along racial and ethnic lines. Am J Clin Nutr. 2020;112(3):566-75.

10 Wells J. Reconsidering the "thin-fat" Indian neonate. J Nutr. 2020;150(4):658-60.

11 Prado CM, Siervo M, Mire E, Heymsfield SB, Stephan BC, Broyles S, et al. A populationbased approach to define body-composition phenotypes. Am J Clin Nutr. 2014;99(6): 1369-77.

12 Siervo M, Prado CM, Mire E, Broyles S, Wells JC, Heymsfield S, et al. Body composition indices of a load-capacity model: gender- and BMI-specific reference curves. Public Health Nutr. 2015;18(7):1245-54. 
13 Plachta-Danielzik S, Gehrke MI, Kehden B, Kromeyer-Hauschild K, Grillenberger $\mathrm{M}$, Willhöft C, et al. Body fat percentiles for German children and adolescents. Obes Facts. 2012;5(1):77-90.

14 Plachta-Danielzik S, Landsberg B, Seiberl J, Gehrke MI, Gose M, Kehden B, et al. Längsschnittdaten der Kieler Adipositas-Präventionsstudie (KOPS). Bundesgesundheitsblatt - Gesundheitsforsch - Gesundheitsschutz. 2012;55(6-7):885-91.

15 Müller MJ, Asbeck I, Mast M, Langnäse K, Grund A. Prevention of obesity - more than an intention. Concept and first results of the Kiel Obesity Prevention Study (KOPS). Int J Obes. 2001;25:S66-74.

16 Plachta-Danielzik S, Landsberg B, Lange D, Langnäse K, Müller MJ. 15 jahre Kieler adipositas-präventionsstudie

(KOPS). Bundesgesundheitsblatt - Gesundheitsforsch - Gesundheitsschutz. 2011 Mar 25;54(3): 304-12.

17 Plachta-Danielzik S, Müller MJ, Bartel C, Raspe H, Thyen U, Landsberg B, et al. Assessment of representativity of a study population - experience of the Kiel obesity prevention study (KOPS). Obes Facts. 2008;1:325-30.

18 Bosy-Westphal A, Schautz B, Later W, Kehayias JJ, Gallagher D, Müller M. What makes a BIA equation unique? Validity of eight-electrode multifrequency BIA to estimate body composition in a healthy adult population. Eur J Clin Nutr. 2013;67:S14-21.

19 Bosy-Westphal A, Jensen B, Braun W, Pourhassan M, Gallagher D, Müller MJ. Quantification of whole-body and segmental skeletal muscle mass using phase-sensitive 8-electrode medical bioelectrical impedance devices. Eur J Clin Nutr. 2017;71(9):1061-7.

20 Cole TJ, Green PJ. Smoothing reference centile curves: the lms method and penalized likelihood. Stat Med. 1992;11(10):1305-19.
21 Wells JC. Toward body composition reference data for infants, children, and adolescents. Adv Nutr. 2014;5(3):320S-9S.

22 Wells J, Davies PSW, Fewtrell MS, Cole TJ. Body composition reference charts for UK infants and children aged 6 weeks to 5 years based on measurement of total body water by isotope dilution. Eur J Clin Nutr. 2020;74(1): 141-8.

23 Cederholm T, Barazzoni R, Austin P, Ballmer P, Biolo G, Bischoff SC, et al. ESPEN guidelines on definitions and terminology of clinical nutrition. Clin Nutr. 2017;36:49-64.

24 Plachta-Danielzik S, Gehrke MI, Kehden B, Kromeyer-Hauschild K, Grillenberger M, Willhöft C, et al. Body fat percentiles for German children and adolescents. Obes Facts. 2012;5(1):77-90.

25 Powell M, Lara J, Mocciaro G, Prado CM, Battezzati A, Leone A, et al. Association between ratio indexes of body composition phenotypes and metabolic risk in Italian adults. Clin Obes. 2016;6(6):365-75.

26 Wells JCK. The capacity-load model of noncommunicable disease risk: understanding the effects of child malnutrition, ethnicity and the social determinants of health. Eur J Clin Nutr. 2018;72(5):688-97.

27 Schutz Y, Kyle UU, Pichard C. Fat-free mass index and fat mass index percentiles in Caucasians aged 18-98 y. Int J Obes Relat Metab Disord. 2002;26:953-60.

28 Stanfield KM, Wells JC, Fewtrell MS, Frost C, Leon DA. Differences in body composition between infants of South Asian and European ancestry: the London mother and baby study. Int J Epidemiol. 2012;41(5):1409-18.

29 Wells JC, Chomtho S, Fewtrell MS. Programming of body composition by early growth and nutrition. Proc Nutr Soc. 2007;66(3): 423-34.
30 Yajnik CS, Fall CH, Coyaji KJ, Hirve SS, Rao S, Barker DJ, et al. Neonatal anthropometry: the thin-fat Indian baby. The Pune maternal nutrition study. Int J Obes Relat Metab Disord. 2003;27(2):173-80.

31 Wells JC, Victora CG. Indices of whole-body and central adiposity for evaluating the metabolic load of obesity. Int J Obes. 2005;29(5): 483-9.

32 von Kries R, Beyerlein A, Müller MJ, Heinrich J, Landsberg B, Bolte G, et al. Different agespecific incidence and remission rates in preschool and primary school suggest need for targeted obesity prevention in childhood. Int J Obes. 2012;36(4):505-10.

33 Whitaker RC, Wright JA, Pepe MS, Seidel KD, Dietz WH. Predicting obesity in young adulthood from childhood and parental obesity. N Engl J Med. 1997;337(13):869-73.

34 Kapoor N. Thin fat obesity: the tropical phenotype of obesity. In: Feingold KR, Anawalt B, Boyce A, Chrousos G, de Herder WW, Dhatariya K, et al., editors. NCBI Bookshelf, Endotext (Internet). South Dartmouth (MA): MDText.com, Inc.; 2021.

35 Schienkiewitz A, Brettschneider A-K, Damerow S, Schaffrath Rosario A. Overweight and obesity among children and adolescents in Germany. Results of the cross-sectional KiGGS wave 2 study and trends. J Heal Monit. 2018;3(1):15-22.

36 Bosy-Westphal A, Jensen B, Braun W, Pourhassan M, Gallagher D, Müller MJ. Quantification of whole-body and segmental skeletal muscle mass using phase-sensitive 8-electrode medical bioelectrical impedance devices. Eur J Clin Nutr. 2017;71(9):1061-7.

37 Bosy-Westphal A, Schautz B, Later W, Kehayias JJ, Gallagher D, Müller MJ. What makes a BIA equation unique? Validity of eight-electrode multifrequency BIA to estimate body composition in a healthy adult population. Eur J Clin Nutr. 2013;67 Suppl 1:S14-21. 